\title{
Measurement and Analysis Plan for Investigation of Spent-Fuel Assay Using Lead Slowing-Down Spectroscopy
}
LE Smith
JJ Ressler
DA Haas
SM Bowyer
Al Gavron ${ }^{1}$
Y Danon ${ }^{2}$
GR Imel $\left.\right|^{3}$
D. Beller ${ }^{4}$

September 2009

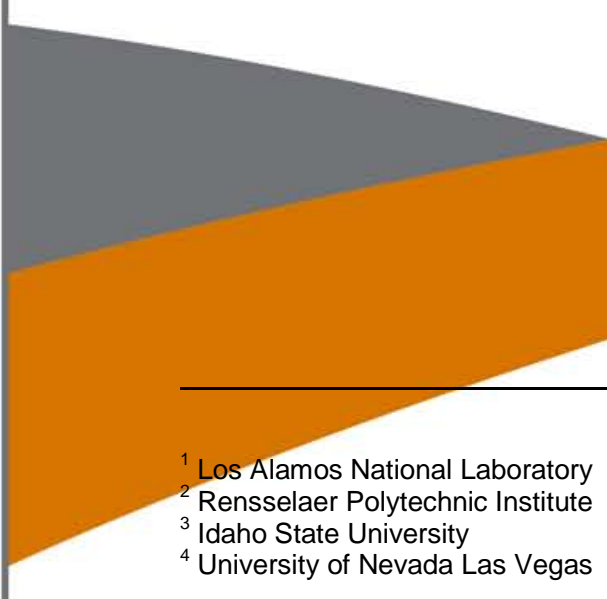

Pacific Northwest NATIONAL LABORATORY 


\section{DISCLAIMER}

This report was prepared as an account of work sponsored by an agency of the United States Government. Neither the United States Government nor any agency thereof, nor Battelle Memorial Institute, nor any of their employees, makes any warranty, express or implied, or assumes any legal liability or responsibility for the accuracy, completeness, or usefulness of any information, apparatus, product, or process disclosed, or represents that its use would not infringe privately owned rights. Reference herein to any specific commercial product, process, or service by trade name, trademark, manufacturer, or otherwise does not necessarily constitute or imply its endorsement, recommendation, or favoring by the United States Government or any agency thereof, or Battelle Memorial Institute. The views and opinions of authors expressed herein do not necessarily state or reflect those of the United States Government or any agency thereof.

PACIFIC NORTHWEST NATIONAL LABORATORY

operated by

BATTELLE

for the

UNITED STATES DEPARTMENT OF ENERGY

under Contract DE-AC05-76RL01830 


\title{
Measurement and Analysis Plan for Investigation of Spent-Fuel Assay Using Lead Slowing-Down Spectroscopy
}

\author{
LE Smith JJ Ressler \\ DA Haas SM Bowyer \\ Al Gavron ${ }^{1} \quad$ Y Danon ${ }^{2}$ \\ GR Imel $^{3} \quad$ D. Beller ${ }^{4}$
}

September 2009

Prepared for the U. S. DEPARTMENT OF ENERGY under Contract DE-AC05-76RL01830

Pacific Northwest National Laboratory Richland, WA 99354

${ }^{1}$ Los Alamos National Laboratory

${ }^{2}$ Rensselaer Polytechnic Institute

${ }^{3}$ Idaho State University

${ }^{4}$ University of Nevada Las Vegas 


\section{Summary}

Under funding from the Department of Energy Office of Nuclear Energy's Material Protection, Accounting, and Control for Transmutation (MPACT) program (formerly the Advanced Fuel Cycle Initiative Safeguards Campaign), Pacific Northwest National Laboratory (PNNL) and Los Alamos National Laboratory (LANL) are collaborating to study the viability of lead slowing-down spectroscopy (LSDS) for spent-fuel assay. Based on the results of previous simulation studies conducted by PNNL and LANL to predict potential LSDS performance, a more comprehensive study of LSDS viability has been defined. That study includes benchmarking measurements, development and testing of key enabling instrumentation, and continued study of time-spectra analysis methods.

This report satisfies the requirements for a PNNL/LANL deliverable that describes the objectives, plans and contributing organizations for a comprehensive three-year study of LSDS for spent-fuel assay. This deliverable was generated largely during the LSDS workshop held on August 25-26, 2009 at Rensselaer Polytechnic Institute (RPI). The workshop itself, also described in this report, was a prominent milestone in the FY09 MPACT project. 


\section{Acronyms}

\begin{tabular}{|c|c|}
\hline AFCI & Advanced Fuel Cycle Initiative \\
\hline AROF & After receipt of funding \\
\hline $\mathrm{D}-\mathrm{T}$ & Deuterium-tritium \\
\hline DOE-NE & United States Department of Energy Office of Nuclear Energy \\
\hline ISU & Idaho State University \\
\hline KAERI & Korea Atomic Energy Research Institute \\
\hline LANL & Los Alamos National Laboratory \\
\hline LANSCE & Los Alamos Neutron Science Center \\
\hline LDRD & Laboratory-Directed Research and Development \\
\hline LINAC & Linear accelerator \\
\hline LSDS & Lead slowing-down spectroscopy \\
\hline MPACT & Material Protection, Accounting, and Control for Transmutation \\
\hline $\mathrm{NE}$ & Office of Nuclear Energy \\
\hline PNNL & Pacific Northwest National Laboratory \\
\hline PSR & Proton storage ring \\
\hline PWR & Pressurized water reactor \\
\hline RPI & Rensselaer Polytechnic Institute \\
\hline SPERT & Special Power Excursion Reactor Test \\
\hline UNLV & University of Nevada Las Vegas \\
\hline US & United States of America \\
\hline WNR & Weapons Neutron Research \\
\hline
\end{tabular}




\section{Contents}

$\begin{array}{lll}1.0 & \text { INTRODUCTION } & 1\end{array}$

$\begin{array}{llr}2.0 & \text { LSDS WORKSHOP } & 1\end{array}$

3.0 BACKGROUND 2

3.1 Key TeChnical Challenges 2

4.0 MEASUREMENT AND ANALYSIS PLAN 3

4.1 OBJECTIVES 3

4.2 ASSUMPTIONS AND PREREQUISITES 3

4.3 SCOPE OVERVIEW $\quad 4$

4.4 PROJECT TASKS 4

4.4.1 Task 1: Modeling and Simulation 4

4.4.2 Task 2: Survey of Pulsed Neutron Sources $\quad 5$

4.4.3 Task 3: Instrumentation Design and Testing: $\quad 5$

4.4.4 Task 4: Empirical Validation $\quad 6$

4.4.5 Task 5: Development, Testing of Time-Spectra Analysis Algorithms 6

4.4.6 Task 6: Coordination with Other LSDS Projects 6

4.4.7 Task 7: Viability Conclusions, Prototype Design Recommendations $\quad 7$

4.5 Milestones AND DeliVERABles $\quad 8$

$\begin{array}{lc}\text { APPENDIX A } & \text { A.1 }\end{array}$

$\begin{array}{lc}\text { APPENDIX B } & \text { B.1 }\end{array}$ 


\section{Figures}

Figure 1. Reporting relationships................................................................... A.2

Figure 2: The Gaerttner LINAC and the Lead Slowing-Down Spectrometer at RPI....... B.2

Figure 3: Layout of LANSCE accelerator complex ................................................. B.3

Figure 4: Schematic drawing of LANSCE Lead Slowing-down Spectrometer .............. B.4 


\subsection{Introduction}

For the last two years, Pacific Northwest National Laboratory (PNNL) and Los Alamos National Laboratory (LANL) have been studying the viability of using lead slowing-down spectroscopy (LSDS) for spent-fuel assay, under funding from the DOE-NE's Materials Protection Accounting and Control for Transmutation (MPACT) program (formerly Advanced Fuel Cycle Initiative Safeguards Campaign). The simulation-based LSDS performance estimates generated by PNNL and LANL to date have encouraged a more comprehensive study of LSDS viability that includes benchmarking measurements, development and testing of key enabling instrumentation, and continued study of time-spectra analysis methods.

A prominent milestone for PNNL and LANL in the FY09 MPACT project was the coordination of an LSDS workshop to help define that more comprehensive study. This report, the PNNL/LANL deliverable, describes the objectives, plans and contributing organizations for the viability study. The workshop was held August 25-26, 2009 at Rensselaer Polytechnic Institute (RPI) and is described in more detail below. This document represents the PNNL/LANL deliverable—a measurements and analysis plan for the proposed three-year study of LSDS for spent-fuel assay.

\subsection{LSDS Workshop}

Attendees represented expertise and experience in several technical areas: LSDS design, physics and measurements for both cross sections and material assay; fission-chamber and alternative neutron detector instrumentation development; modeling and simulation; and time-spectra analysis methods. These attendees also represented the organizations that will form the LSDS collaboration in the MPACT program.

- PNNL: Eric Smith, Jennifer Ressler, Sonya Bowyer, Derek Haas

- LANL: Victor Gavron

- RPI: Yaron Danon, Robert Block, Devin Barry, Jeff Hoole, Cathy Romano

- Idaho State University: George Imel

- University of Nevada Las Vegas: Denis Beller (unable to attend workshop)

The primary objectives of the workshop were:

1. Technical exchange among LSDS practitioners;

2. Identification of key challenges in the application of LSDS to fuel assay;

3. Refinement of scope and organizational roles for an FY10-12 project;

4. Identification of linkages to LSDS-related activities and projects outside MPACT. 
The workshop was successful in meeting all of these objectives, as evidenced by the contents of this document. The measurement and analysis plan presented here was primarily generated from the discussions that occurred during the workshop, but follow-up discussion and review comments aided the completion of this document.

\subsection{Background}

The direct measurement of Pu in spent-fuel assemblies is important to the safeguarding of domestic fuel cycles in ways that include plutonium input accountability (prior to processing spent fuel), shipperreceiver confirmation, and burnup credit prior to repository storage. LSDS is a spent-fuel assay technique that has the potential to meet these objectives by directly measuring $\mathrm{Pu}$ isotopic mass (i.e., ${ }^{239} \mathrm{Pu}+{ }^{240} \mathrm{Pu}+$ ${ }^{241} \mathrm{Pu}$ ) in an irradiated assembly, while simultaneously measuring other fissile isotopes such as ${ }^{235} \mathrm{U}$. Previous modeling and analysis by this team has indicated that Pu uncertainties of less than $5 \%$ are achievable and that lower values may be possible with improved time-spectra analysis techniques and advanced instrumentation.

FY08-FY09 evaluations of LSDS potential in the MPACT program have been based on simulated fuel assay with optimistic assumptions about pulsed-neutron source intensity, the availability and efficacy of fission-chamber technologies, and the impact of potentially reducing backgrounds. To more definitively assess the value of LSDS for safeguards applications, it is important to include more realistic assumptions in the viability analysis and to move the LSDS evaluation beyond simulations to empirical validation using representative instruments and samples.

\subsection{Key Technical Challenges}

The workshop collaborators have identified the following as key technical challenges for LSDS assay of spent fuel:

1. Study the effects of self-shielding using simulation and measurement, and develop time-spectra analysis methods that can overcome that effect to produce accurate isotopic assay over a range of fuel types and burnups;

2. Perform empirical benchmarking measurements of LSDS physics parameters and representative U and Pu samples, to build confidence in simulation-based performance predictions for fuels that cannot be measured at this time;

3. Develop threshold neutron detector alternatives that do not require ultra-pure ${ }^{238} \mathrm{U}$ and have substantially higher intrinsic efficiency than threshold fission chambers can provide;

4. Identify appropriate pulsed-neutron source options and perform analysis to support design recommendations. 


\subsection{MPACT LSDS Project Measurement and Analysis Plan}

\subsection{Objectives}

The overall goal of the FY10-FY12 project is to complete a comprehensive assessment of the viability of LSDS for the direct measurement of Pu (and other) isotopes in spent-fuel assemblies. The three-year effort described here will focus on the following objectives:

1. Enabling Instrumentation: Develop and test the instrumentation suite (e.g., threshold and fissile fission chambers) required to support LSDS fuel assay measurements;

2. Benchmarking Measurements: Benchmark modeling predictions for spent fuel using measurements on representative fresh fuel (single pins and assemblies) at the RPI LSDS facilities;

3. Time-Spectra Analysis Algorithms: Continue the development and refinement of time-spectra analysis methods that extract isotopic mass from the complex LSDS assay signal;

4. Performance Predictions: Quantitatively predict, using high-fidelity simulations and suitable signal analysis methods, the performance of LSDS for the assay of pressurized water reactor (PWR) fuel assemblies over a range of initial composition, burnup and cooling times;

5. Viability and Design Recommendations: If the three-year project indicates LSDS viability for spentfuel assay, a set of design recommendations (e.g., pulsed neutron source specifications, physical design of lead moderator, instrumentation types, and fuel handling options) will be drafted to support the potential development of a fuel-assay prototype system.

\subsection{Assumptions and Prerequisites}

Key assumptions in this work include the availability of the high- and ultra-high-purity materials needed to fabricate an array of fissile (e.g., ${ }^{239} \mathrm{Pu}$ and ${ }^{241} \mathrm{Pu}$ ) and threshold fission chambers (e.g., ${ }^{238} \mathrm{U}$ or ${ }^{232} \mathrm{Th}$ ) to utilize at the RPI and Los Alamos Neutron Science Center (LANSCE) LSDS facilities. It is assumed that a sufficient quantity and purity of ${ }^{238} \mathrm{U}$ can be obtained from the University of Nevada Las Vegas (UNLV). However, if this is not the case, the threshold chambers may present the greatest challenge due to the difficulty in procuring (within reasonable cost and schedule) an alternate source of ultra-pure ${ }^{238} \mathrm{U}$. Therefore, alternative threshold materials (e.g., ${ }^{232} \mathrm{Th}$ and ${ }^{237} \mathrm{~Np}$ ) will be considered and existing U.S. inventories of materials such as those used to support experiments at the Zero Power Physics Reactor will be leveraged as much as possible.

A second key assumption is the availability and operability of existing LSDS facilities at LANL and RPI. Completion of preliminary benchmarking measurements for the earliest prototype fission chambers is dependent on the availability of beam time at the LANSCE LSDS facility at LANL, and those measurements are currently scheduled for November, 2009. Funding for RPI in FY10 will support their restart and re-characterization of the LSDS instrument, an instrument that should provide greater 
flexibility in the availability of beam time and the samples to be used for benchmarking (e.g., a section of a Special Power Excursion Reactor Test-SPERT—fresh fuel assembly). Completion of the final benchmarking measurements at RPI is dependent on the availability of Pu foils to wrap around the fresh SPERT fuel, as a means to emulate high-burnup spent fuel. The source of those foils is yet to be determined, as are the cost and time associated with sending them to RPI.

\subsection{Scope Overview}

The FY10-FY12 project described here will be executed by a collaboration that has been established and growing since FY08. This collaboration includes PNNL, LANL, RPI, Idaho State University (ISU) and University of Nevada Las Vegas (UNLV). More detailed information on the collaboration and associated program logistics is presented in Appendix A and a description of the key measurement facilities is provided in Appendix B. An overview of the role of each collaborator is presented in the table below. Specific tasks are detailed in Section 4.4 and the Milestones and Deliverables are presented in Section 4.5

\begin{tabular}{|c|l|}
\hline Institution & \multicolumn{1}{|c|}{ Role } \\
\hline PNNL & $\begin{array}{l}\text { Collaboration and inter-program coordination, modeling and simulation, time-spectra } \\
\text { analysis, pulsed-neutron source survey and analysis }\end{array}$ \\
\hline LANL & $\begin{array}{l}\text { Fission-chamber characterization measurements at LANL, modeling and simulation, time- } \\
\text { spectra analysis methods }\end{array}$ \\
\hline RPI & $\begin{array}{l}\text { Benchmarking measurements of LSDS physics parameters and fresh fuel (single pin and } \\
\text { full assemblies), alternative detector options }\end{array}$ \\
\hline ISU & $\begin{array}{l}\text { Fission-chamber design and testing methods, linkages to high-purity fission-chamber } \\
\text { materials }\end{array}$ \\
\hline UNLV & Characterization and refinement of ultra-pure ${ }^{238} \mathrm{U}$ chambers \\
\hline
\end{tabular}

\subsection{Project Tasks}

\subsubsection{Task 1: Modeling and Simulation}

This task will leverage modeling tools (e.g., ORIGEN and MCNP) developed under previous Advanced Fuel Cycle Initiative (AFCI) funding, but will extend the simulations to include a detailed model of the RPI LSDS that will be utilized for the benchmarking measurements. This task will also include modeling of specific neutron sensor designs, for example fission chamber variants or alternative neutron detector ideas. This modeling will support concept viability studies as well as design optimization work for the most promising detector concepts. Pulsed-neutron sources will also be simulated to better understand the pros and cons of various types (e.g., electron versus proton accelerators) in terms of pulse intensity, 
gamma-ray flash intensity, neutron energy and temporal distributions, etc. All of the modeling, ultimately, will be aimed to support design recommendations (e.g., requirements on pulsed-neutron intensity) based on these realistic performance predictions for LSDS as applied to PWR assemblies over a wide range of initial composition, burnup and cooling times. This DOE-NE project will leverage, as much as possible, the MCNP spent-fuel library already being developed under NA-24 funding.

\subsubsection{Task 2: Survey of Pulsed Neutron Sources}

Previous analysis has indicated that air-cooled deuterium-tritium (D-T) pulsed neutron generator are not sufficiently intense to overcome the neutron background in high burnup PWR fuels. While this preliminary assessment is not conclusive, it does encourage the exploration of other pulsed-neutron source options for their applicability in LSDS assay. Both electron and proton accelerators are possibilities. For electron accelerators, options include low-energy accelerators ( $<15 \mathrm{MeV}$ is preferred due to substantially lower cost) and advanced target designs using Be or D for enhanced photo-neutron production. Higher energy ( 40 MeV) electron accelerators require moderate electron current $(\sim 20 \mu \mathrm{A})$ in order to achieve the desired yield and will also be considered. Recent advances in highly compact and significantly less expensive proton accelerators (driven primarily by the medical community's demand for superior and more readily available proton radiation treatment facilities) will be exploited to provide potential proton-based pulsed neutron sources. Parameters to consider for either option include achievable neutron pulse width, neutron pulse intensity, neutron energy distribution, photon-flash intensity and energy spectrum, operating complexity, size, and approximate cost. The survey will help support viability and prototype design recommendations, and will also feed into the modeling and simulation task described above.

\subsubsection{Task 3: Instrumentation Design and Testing:}

The fission chambers utilized in LSDS measurements present considerably different requirements than the reactor instrumentation that drives commercial offerings and consequently, a concerted effort to design, fabricate and test fission chambers tailored for LSDS is required. The electrodeposition techniques utilized in fission-chamber fabrication are nearly a lost art in the DOE community and will need to be revived in this task. Additionally, the high-purity materials (e.g., ultra-pure ${ }^{238} \mathrm{U},{ }^{235} \mathrm{U},{ }^{239} \mathrm{Pu}$ and ${ }^{241} \mathrm{Pu}$ ) are no longer as readily available as they once were, making the collection or procurement of these materials a notable challenge. Previous analysis has indicated that a key limiting factor in LSDS assay is the very low intrinsic efficiency of threshold fission chambers used to collect the induced neutron assay signal. Study into alternative threshold detector techniques in this task will be coordinated with related work in non-MPACT projects. Various instrumentation options will be studied and prototypes will be built and tested in the high-fluence LSDS system at LANL's LANSCE or at RPI. Initial testing also can be performed at ISU. For validation and comparison, the ultra-pure, ultra-depleted ${ }^{238} \mathrm{U}$ that is now on-hand at UNLV is intended to be used in simple fission chambers. 


\subsubsection{Task 4: Empirical Validation}

The Gaerttner Linear Accelerator (LINAC) Laboratory at RPI has an LSDS instrument that will be used to perform basic LSDS system characterization measurements, as well as single-pin and full-assembly measurements. A 4\%-enriched, unirradiated light-water reactor fuel assembly (SPERT) will be the cornerstone of the campaign. To the degree possible, environments typical of irradiated fuel assay will also be created. For example, intense gamma-ray and spontaneous fission neutron sources will be combined with RPI's fresh fuel samples in the interrogation chamber to emulate the fission-product and

${ }^{244} \mathrm{Cm}$ emissions in high-burnup fuels.

The LANSCE facility at LANL will be a second location for empirical validation and instrumentation testing. LANSCE is currently authorized to study samples of plutonium and uranium and has in its inventory low-mass fissile isotope samples (a few grams each), including ${ }^{239} \mathrm{Pu}$ that is not available at RPI. The LANL LSDS is driven by intense neutron pulses generated by the LANSCE $800-\mathrm{MeV}$ proton LINAC, and compressed to narrow pulse widths (less than 270 nanoseconds) suitable for LSDS. This high-fluence environment will support fission-chamber design and testing work by ISU and UNLV.

In combination, the SPERT measurements at RPI and the low-mass-sample measurements at LANL will provide ${ }^{235} \mathrm{U}$ and ${ }^{239} \mathrm{Pu}$ measurement data that can be compared to MCNP simulations of the same configurations, thereby providing confidence in the simulations used to predict full-assembly performance with more complex fuel matrices.

\subsubsection{Task 5: Development and Testing of Time-Spectra Analysis Algorithms}

Previous studies by PNNL and LANL have shown that signal analysis methods to quantify isotopic mass from the assay signal can have a significant impact on the achievable accuracy and precision of LSDS assay. The focus of this task will be developing time-spectra methods that require no training or calibration, and therefore, can achieve truly direct measurement of Pu in spent fuel assemblies. However, supervised learning algorithms will also be considered for the scenario in which well-characterized spent fuel assemblies could be used to train for subsequent assays. The time-spectra analysis method development will be founded on simulated full-assembly assay signals that mimic the complicating selfshielding effects of realistic fuel assay. The signal processing algorithms will also be applied to the single-pin and full-assembly measured assay data to assess the impacts of empirical systematic effects.

\subsubsection{Task 6: Coordination with Other LSDS Projects}

As described below, the MPACT LSDS project is the centerpiece of LSDS study and development in the United States. However, there are two other projects that will provide complementary technical support and evaluation: NA-24's Spent-fuel assay Evaluation project and PNNL's internally-funded project to build an LSDS test bed dedicated to safeguards applications and to develop enabling neutron detector instrumentation. From the former, this NE project will leverage the LANL-developed MCNP fuel 
assembly library. Methods and metrics for evaluating fuel assay techniques, as they are defined in the NA-24 study, will be utilized (as appropriate) for this NE study. The internally-funded PNNL project (funded through Laboratory-Directed Research and Development [LDRD]) will study exploratory concepts for neutron detection instrumentation; promising concepts could then be evaluated in the RPI or LANSCE LSDS facilities as a part of this NE project's instrumentation assessment. See Appendix A for further discussion of inter-program connections.

\subsubsection{Task 7: Viability Conclusions and Prototype Design Recommendations}

If the three-year study described here results in a positive viability conclusion for LSDS assay of spent fuel, prototype design recommendations will be formulated based on lessons learned. These recommendations would include specifications for the pulsed neutron source, lead moderator design, instrumentation specifications, concepts for fuel handling, etc., and would provide the foundation for a follow-on project to site and build a prototype LSDS at a fuel handling facility. 


\subsection{Milestones and Deliverables}

The three-year Milestones (M) and Deliverables (D) schedule for the project is presented below. Dates are indicated as months after receipt of funding (AROF) this list also assumes that proposed funding levels are realized. Different funding levels and/or findings in the first years of the project may require modification of scope, schedule or both.

\begin{tabular}{|c|l|c|l|}
\hline $\begin{array}{c}\text { Months } \\
\text { (AROF) }\end{array}$ & \multicolumn{1}{|c|}{ Description } & Type & \multicolumn{1}{|c|}{ Leads } \\
\hline 3 & Complete cross-comparison of absolute LSDS count rates and statistical noise & M & PNNL, LANL \\
\hline 6 & Complete survey of pulsed neutron source options & M & PNNL, LANL \\
\hline 6 & $\begin{array}{l}\text { Complete measurements with prototype }{ }^{232} \text { Th chamber at the Los Alamos } \\
\text { National Science Center (LANSCE) }\end{array}$ & M & LANL \\
\hline 8 & $\begin{array}{l}\text { Complete detailed MCNP model of RPI LSDS and benchmark using previous } \\
\text { measurements of physics parameters material assay }\end{array}$ & M & RPI, PNNL \\
\hline 12 & $\begin{array}{l}\text { Complete initial comparative study of time-spectra analysis algorithms (direct } \\
\text { and supervised) using MCNP fuel library developed by NA-24 }\end{array}$ & M & PNNL \\
\hline 12 & $\begin{array}{l}\text { Complete fabrication and initial testing of threshold fission chamber using ultra- } \\
\text { pure }{ }^{238} \text { U from Russian supplier }\end{array}$ & M & ISU, UNLV \\
\hline 12 & Technical report summarizing methods, issues and findings to date & D & All \\
\hline 15 & Complete empirical assay of single SPERT fuel pin, compared to simulations & M & RPI, PNNL \\
\hline 15 & Assay small (gram-level) U and Pu samples at LANSCE & M & LANL \\
\hline 24 & Complete study and testing of neutron detection options & M & ISU \\
\hline 24 & Complete empirical assay of 8x8 SPERT assembly, compare to simulated & M & RPI, PNNL \\
\hline 24 & Technical report summarizing methods, issues and findings to date & D & ALL \\
\hline 30 & $\begin{array}{l}\text { Complete empirical assay of 8x8 SPERT assembly with Pu foils, compare to } \\
\text { simulated }\end{array}$ & M & RPI, PNNL \\
\hline 36 & Complete LSDS viability study and draft recommendations & M & All \\
\hline 36 & $\begin{array}{l}\text { Final report summarizing findings and as appropriate, LSDS prototype design } \\
\text { recommendations }\end{array}$ & All \\
\hline & DA & \\
\hline
\end{tabular}




\section{Appendix A}

\section{LSDS Collaboration Coordination}

This appendix provides supporting information for the LSDS Measurement and Analysis Plan and includes a detailed description of the collaboration and the collaboration coordination that enable this work. Also described are the programmatic connections between this DOE-NE project and other LSDSrelated projects

\section{A.1 LSDS Collaboration}

A proper balance of simulation and benchmarking measurements requires the collaborative effort of the organizations who have studied LSDS in the past. Each of the organizations involved in this project were chosen for their particular experience, expertise and facilities. The complementary strengths of the various institutions are notable and have been elucidated in this plan. No single organization has the staff, facilities and expertise needed to meet the project objectives, but the team does.

The proposed funding level for this project is significant, reflecting the various facets, both computational and experimental, required to achieve the objective of comprehensively evaluating LSDS viability for spent-fuel assay. The operation and use of the experimental facilities at RPI and LANL is an expensive endeavor, but absolutely crucial to building confidence in and demonstrating the accuracy of the simulation methods used to predict full-assembly performance. The management and coordination of this effort, which spans five different organizations, will also require significant resources.

The heavy involvement of universities in this project, and the support of graduate students and faculty engaged in the evaluation of LSDS, will serve as a conduit in further establishing U.S. expertise and experience in the area of nuclear fuel cycle safeguards. The use of the experimental facilities at RPI and LANL will leverage previous infrastructure investment (U.S. Government and other sources), while aiming at a recognized safeguards need.

\section{Coordination and Management Plan}

- Process for setting technical direction: Monthly teleconferences and collaboration notes will document outstanding issues, their resolution, and progress against milestones. Quarterly meetings hosted by the collaborating organizations will also be key in setting technical direction and informing the team.

- Publications: Publications will be jointly issued, as appropriate for the subject matter. Lead authorship will be defined by the collaborative team. 
- Intellectual Property Issues: No IP issues are expected to arise in this project, but applicable policies and practices at the participating organizations will be utilized as appropriate.

\section{A.2 Programmatic Connections}

Pictorial representations of the programmatic landscape for LSDS development are given in the graphics below. Connections to the LSDS effort in South Korea at the Korea Atomic Energy Research Institute (KAERI) are yet to be defined.

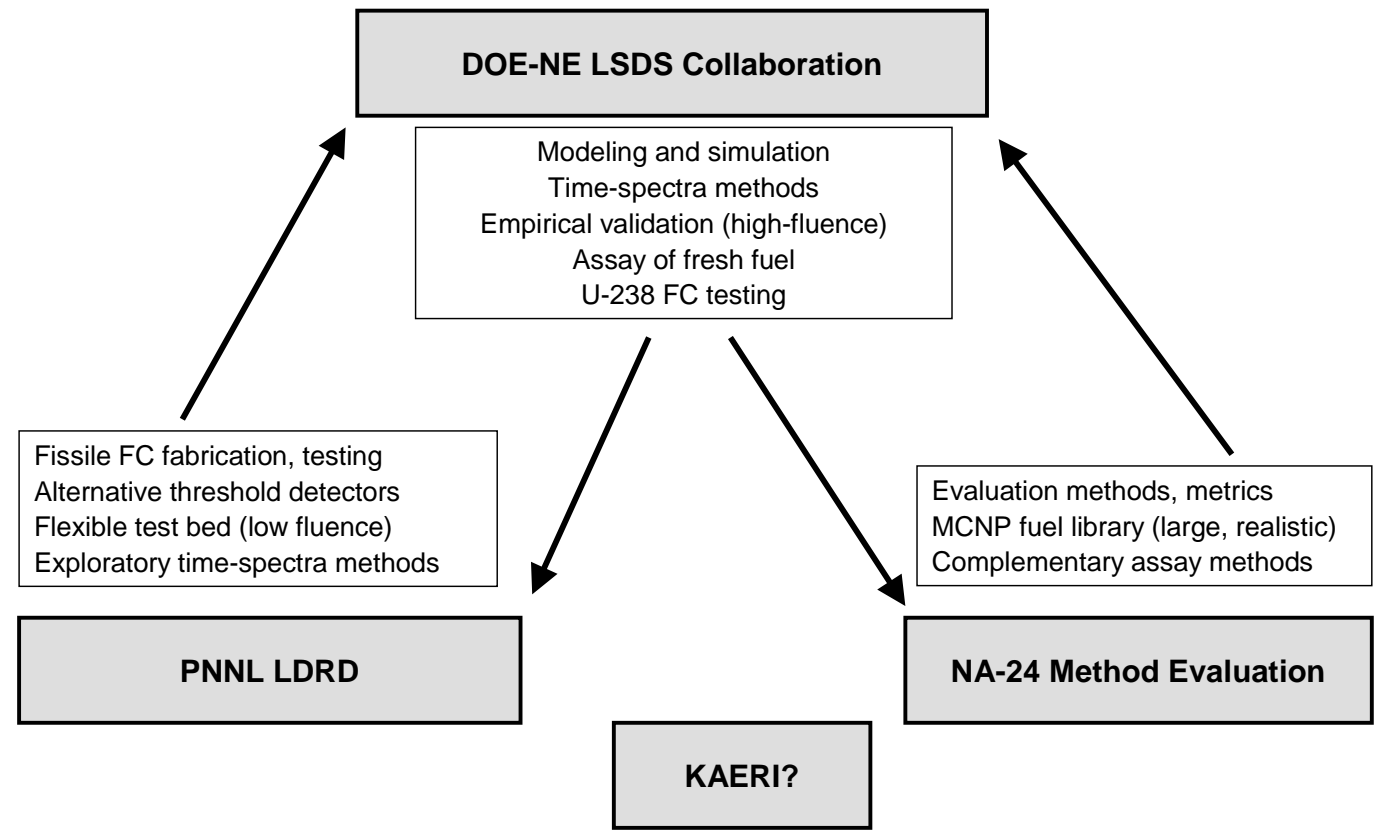

Figure 1. Schematic of scope and relationships for the various program area supporting LSDS research. 


\section{Appendix B}

\section{LSDS Facilities}

This appendix provides a description of the key facilities that will be utilized throughout the LSDS project.

\section{B.1 Key Facilities}

\section{B.1.1 The Gaerttner LINAC Laboratory at Rensselaer Polytechnic Institute}

The Gaerttner LINAC Laboratory is a major facility at Rensselaer Polytechnic Institute (RPI) that has been actively involved in nuclear physics research for over 45 years. It has a world-class reputation in neutron interactions research and, through extensive and continuing upgrading, remains a powerful and modern research facility. The majority of the research funding related to its LINAC is for nuclear data measurements. The LINAC short pulse width and variety of flight paths enable accurate cross section measurements in the resonance and thermal energy regions. Its total neutron production is about $10^{13} \mathrm{n}$ /sec. Its capabilities include ${ }^{6} \mathrm{Li}$-glass detectors, a 16-segment high efficiency multiplicity detector for capture measurements and a lead slowing-down spectrometer currently used for fission cross section measurements of small samples. New capabilities include a neutron scattering detector array coupled to a digital data acquisition system and a new modular large liquid scintillator for transmission measurements with MeV neutrons. Over the years the RPI LINAC research has produced over 160 graduate students. Currently there are six graduate students and two undergraduate students that are performing research with the LINAC. The LINAC is one of a few university facilities that can train nuclear engineers in the area of nuclear data. 

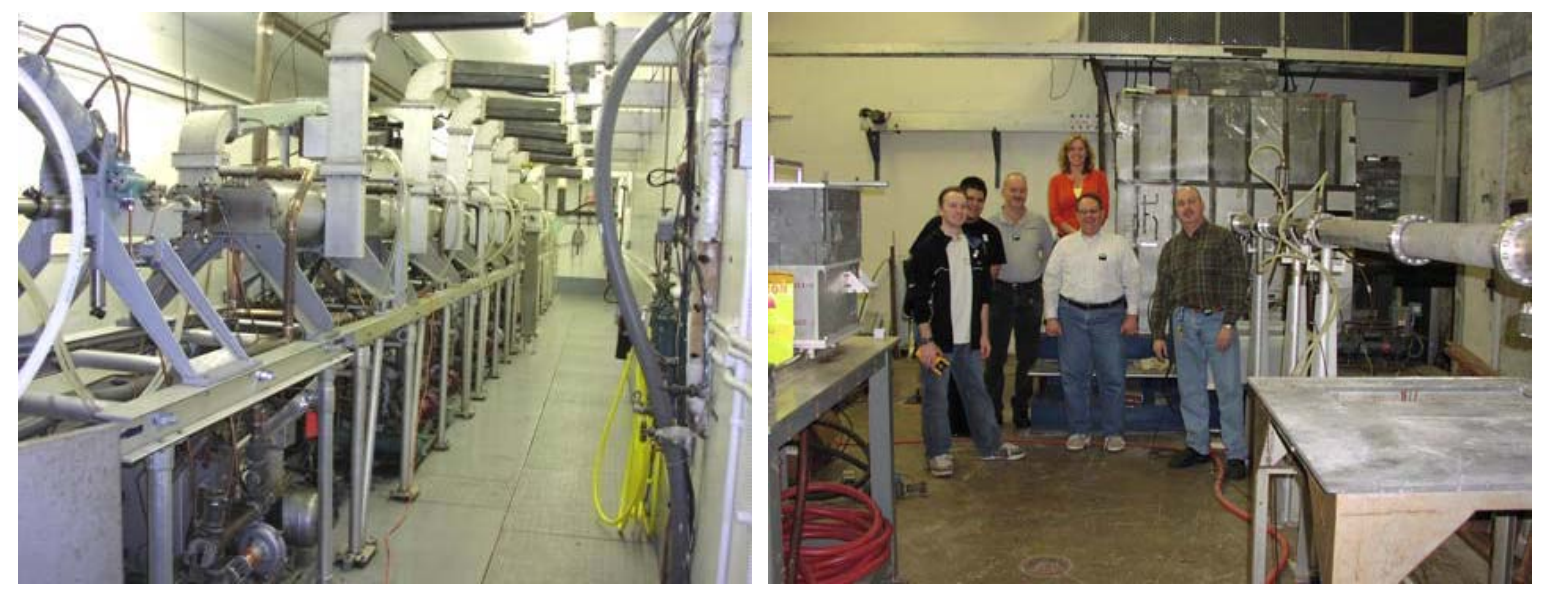

Figure 2: The Gaerttner LINAC at RPI (left) and the Lead Slowing-Down Spectrometer (right)

\section{B.1.2 The LANSCE Facility at Los Alamos National Laboratory}

The heart of the LANSCE facility is a highly flexible LINAC system, one of the most powerful in the world, that can accelerate up to mA of protons to an energy of $800 \mathrm{MeV}$ and then deliver the protons to multiple experimental areas. The LINAC can also accelerate negative hydrogen ions to $800 \mathrm{MeV}$. Protons at $100 \mathrm{MeV}$ are used at the Isotope Production Facility for making medical and other short-lived radioisotopes. Pulses of $800-\mathrm{MeV}$ negative hydrogen ions are used at the Proton Radiography Facility for imaging dynamic events related to nuclear weapons performance and are also sent to heavy-metal targets at the Weapons Neutron Research (WNR) Facility. Proton-nucleus collisions in the targets generate large numbers of neutrons $(\sim 20 \mathrm{n} / \mathrm{p})$ through a process called nuclear spallation. The neutron pulses, in turn, are used for materials irradiation and fundamental and applied nuclear physics research. The negative hydrogen ions are also injected into a 30-m diameter Proton Storage Ring (PSR). The PSR converts a $625 \mu$ s pulse of negative hydrogen ions into an intense 125-ns burst of protons. Those intense proton bursts produce, through nuclear spallation, short bursts of neutrons for neutron scattering studies of material properties at the Lujan Center and for nuclear physics research at the WNR Facility. In addition, a newly commissioned ultracold-neutron research facility is beginning the exploration of fundamental nuclear physics with experiments designed to test the standard model of elementary particles. 


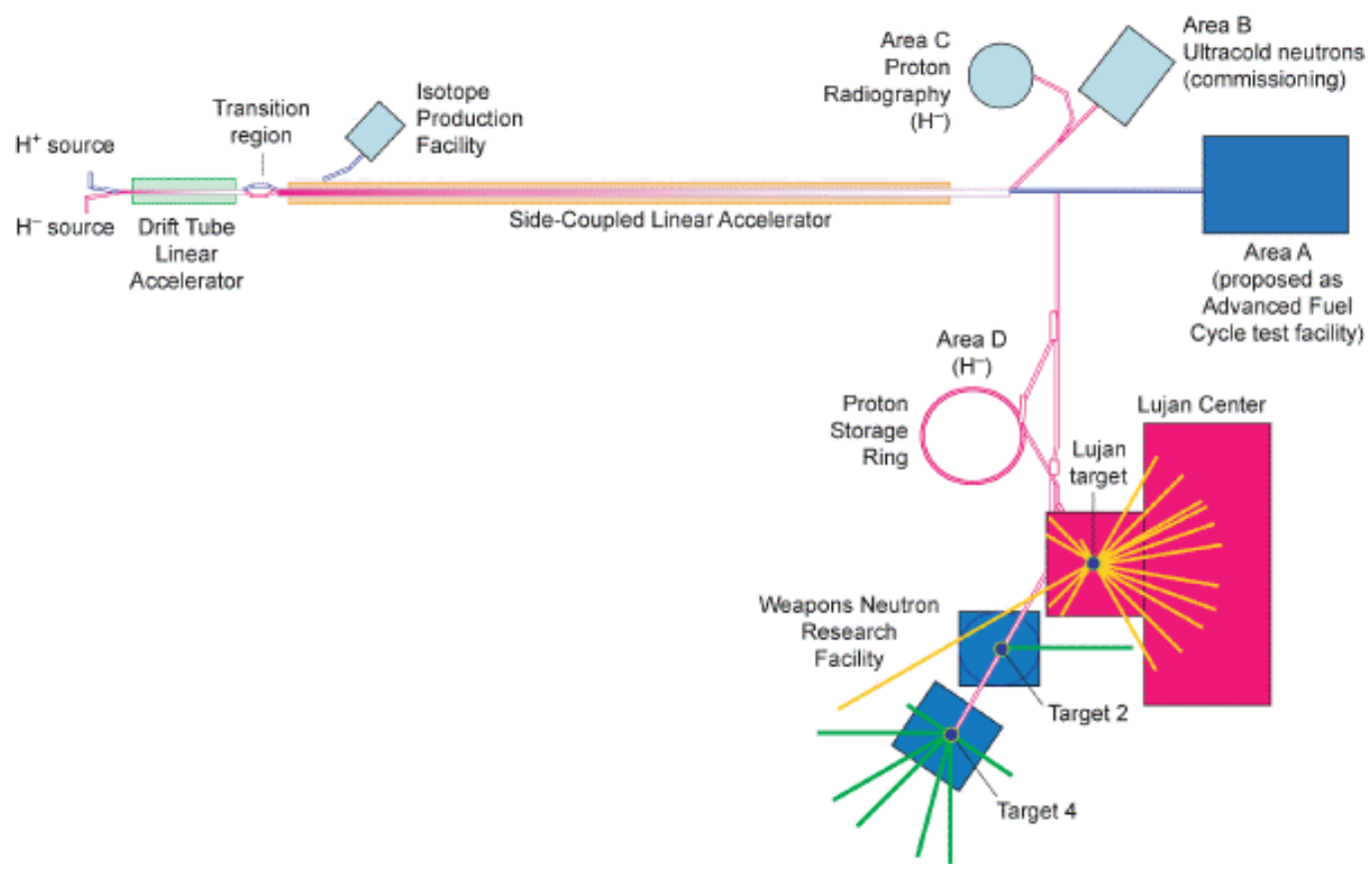

Figure 3: Layout of LANSCE accelerator complex

The Lead Slowing Down Spectrometer (LSDS) that will be used for testing in this project is located in the “Target 2" area. Proton beam pulses ( $800 \mathrm{MeV}, 270$ nanoseconds wide) impinging on a small tungsten target in the middle of a lead cube, produce neutron pulses each of more than $10^{14}$ neutrons per pulse. These neutrons scatter around in the lead cube, losing energy, with a well-known correlation between the elapsed time and energy.

The cube, comprised of many lead blocks, is 1.2 meters on each side. There is a tungsten beam-stop in its center to maximize the production of neutrons. There are additional cavities inside the cube for samples and detectors. The facility possesses samples containing a few grams of ${ }^{239} \mathrm{Pu}$ and ${ }^{235} \mathrm{U}$ that will be inserted in one of these cavities. Fission chambers, lined with both threshold (e.g., ${ }^{238} \mathrm{U}$ ) and fissile (e.g., ${ }^{239} \mathrm{Pu}$ ) materials, will be placed in an adjacent cavity. The number of detected neutrons as a function of the time after the proton pulse generates a signal that will be analyzed and compared to simulated results. 
PNNL-18795

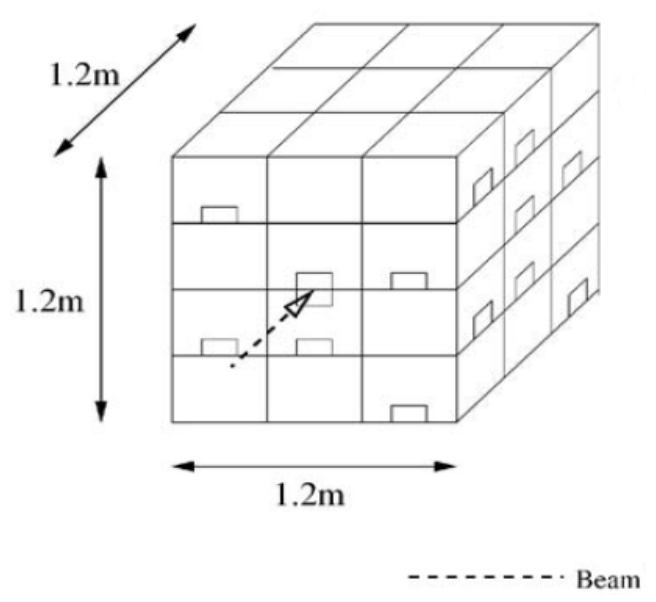

Figure 4: Schematic drawing of LANSCE Lead Slowing-down Spectrometer 


\section{Distribution}

1 Michael Miller

Los Alamos National Laboratory

P.O. Box 1663, MS-H816

Los Alamos, NM 87545

1 Mark Mullen

Los Alamos National Laboratory

P.O. Box 1663, MS-E541

Los Alamos, NM 87545

$1 \quad$ Bradley Williams

NE-54/Germantown Building

U.S. Department of Energy

1000 Independence Avenue, SW

Washington, DC 20585-1290

1 Victor Gavron

Los Alamos National Laboratory

P.O. Box 1663, MS-H845

Los Alamos, NM 87545
1 Yaron Danon

Dept of Mechanical Aerospace and

Nuclear Engineering

NES 1-9

Rensselaer Polytechnic Institute $1108^{\text {th }}$ Street

Troy, NY 12180-3590

1 George Imel

Idaho State University

Nuclear Engineering Dept

921 S. $8^{\text {th }}$ Avenue, Stop 8060

Pocatello, ID 83209-8060

$1 \quad$ Denis Beller

Dept. of Mechanical Engineering

University of Nevada Las Vegas

4505 S. Maryland Parkway

Las Vegas, NV 89154

\section{\# Local Distribution}

Pacific Northwest National Laboratory

1 JL Buelt K2-01

1 LE Smith P8-20

1 JJ Ressler K5-25

1 DA Haas P8-20

1 SM Bowyer P8-20 


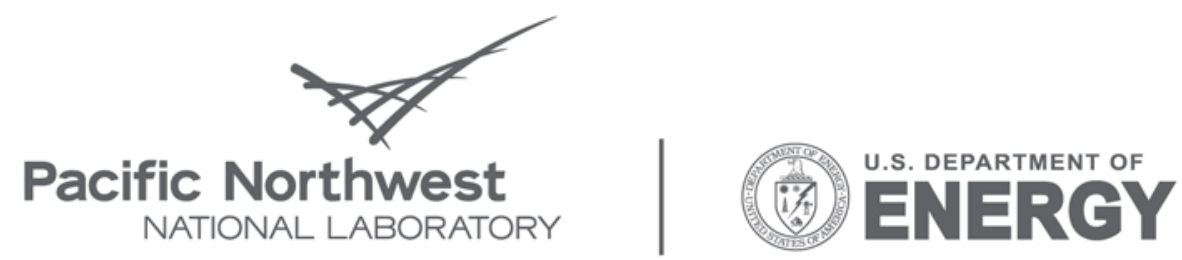

Proudly Operated by Battelle Since 1965

902 Battelle Boulevard

P.O. Box 999

Richland, WA 99352

1-888-375-PNNL (7665)

www.pnl.gov 